\title{
Interferencia entre el procesamiento de estímulos gustativos y estímulos visuales lingüísticos incompletos en la memoria operativa
}

\section{Processing Interference Between Gustative Stimuli and Incomplete Linguistic Visual Stimuli in Working Memory}

Recibido: Marzo de 2013

Revisado: Mayo de 2013

Aprobado: Junio de 2013
Eugenia Razumiejczyk, Guillermo Macbeth, Consejo Nacional de Investigaciones Científicas y Técnicas - Universidad Nacional de Entre Ríos, Argentina

\section{Nora Leibovich de Figueroa}

Consejo Nacional de Investigaciones Científicas y Técnicas - Universidad de Buenos Aires, Argentina

Información de Contacto: Dra. Eugenia Razumiejczyk. Consejo Nacional de Investigaciones Científicas y Técnicas (CONICET). Universidad Nacional de Entre Ríos, Facultad de Ciencias de la Educación (UNER-FCE). Alameda de la Federación 106, E3100GNO, Paraná, Entre Ríos, Argentina.Email: eugeniaraz@ hotmail.com. Teléfono +54343422 2033.

Reconocimientos

La investigación informada en el presente trabajo recibió financiación del Consejo Nacional de Investigaciones Científicas y Técnicas (CONICET) de Argentina mediante el proyecto de investigación plurianual PIP IU No 11420100100139 concedido al primer autor

\section{Resumen}

El propósito de este estudio consiste en extender la evidencia disponible sobre el efecto stroop al cruce de las modalidades gustativa y visual por palabras incompletas. Este fenómeno es indicativo de cierta interferencia en la memoria operativa para la información proveniente de diferentes canales sensoriales. En estudios previos del mismo paradigma se encontró menor interferencia para la congruencia entre los diferentes canales y mayor interferencia para la incongruencia. Los hallazgos previos se extienden por el presente estudio a las modalidades gustativa y visual para estímulos lingüísticos codificados como palabras escritas con letras omitidas. Los resultados sugieren que el mismo 
of this contribution are presented as well as and some suggestions for future research.

Key words: Stroop Effect, Operative Memory, Taste, Vision.

El ser humano se desenvuelve en un medio complejo y estructurado a la vez. La memoria operativa, sistema que mantiene y manipula la información de manera temporal (Baddeley, 1995), permite al individuo manejarse en tal ambiente porque presenta suficientes constantes que le permiten utilizar el pasado como predictor del futuro. Así, el organismo percibe algunos aspectos del ambiente en el que vive apoyándose en regularidades del entorno. La información obtenida a través de diversos canales sensoriales tiene una alta probabilidad de estar vinculada. En este sentido los objetos, además de poseer características tanto visuales como espaciales, tienen aspectos táctiles, olfatorios y gustativos asociados. Por lo tanto, la percepción y la integración de diferentes canales de información se benefician con alguna forma de almacenaje transitorio que permita un procesamiento más prolongado que atienda a los distintos tiempos requeridos por el sistema cognitivo. Esta tarea de integración le corresponde a la memoria operativa (Baddeley, 1995).

$\mathrm{Al}$ respecto, Stroop (1935) realizó tempranamente un estudio en el que administraba palabras cuyos significados aludían a colores. Estas palabras estaban escritas en tintas iguales o diferentes a las que aludían las palabras. La consigna requería nombrar el color con el que estaba escrita la palabra en el menor tiempo posible. Los resultados mostraron que cuando los estímulos eran congruentes (color de tinta color escrito) se registraban mayores aciertos y menores tiempos de reacción (MacLeod, 1991). En oposición, cuando los estímulos eran incongruentes, los participantes presentaban significativamente mayor tiempo de reacción en sus respuestas. La congruencia remite en este contexto a la coherencia semántica entre el color del estímulo y el significado de la palabra. Resulta así congruente la palabra rojo cuando se presenta escrita como estímulo visual en color rojo. Se ha explicado este fenómeno "stroop" como un efecto de competencia atencional entre estímulos (Cho, efecto de interferencia stroop hallado en estudios previos se presenta también en las modalidades específicas aquí estudiadas. Se destaca la condición ecológica del presente aporte por su utilización de estímulos naturales. Se discuten las limitaciones y se proponen nuevos experimentos.

Palabras clave: efecto stroop, memoria de trabajo, gusto, visión.

Lien \& Proctor, 2006; Kahneman \& Chajczyk, 1983; Kim, Cho, Yamaguchi \& Proctor, 2008; Mitterer, LaHeij \& Van der Heijden, 2003). En la tarea experimental solicitada se deben inhibir distractores para poder cumplir con el procesamiento que la prueba requiere (Kirn, Kirn \& Chun, 2005; Sreenivasan \& Jha, 2007). Así, la prueba evalúa aspectos ejecutivos del control intencional requerido por la atención selectiva (Banich et al., 2000; MacLeod, 1991, 1992). La interferencia stroop se produce cuando la función ejecutiva de la atención falla debido a los distractores que son incongruentes con la respuesta que se espera y, por lo tanto, se produce un mayor tiempo de reacción o se cometen más errores en las respuestas. El fenómeno stroop se ha estudiado en diversas situaciones intermodales relacionadas con la percepción gustativa. Se dispone de evidencia experimental para la combinación entre el olfato y el gusto (Prescott, Johnstone \& Francis, 2004; Rolls, 2004; Stevenson \& Boakes, 2004; White \& Prescott, 2007), entre la audición y el gusto (Razumiejczyk, Jáuregui \& Macbeth, 2012) y también entre la visión y el gusto (Razumiejczyk, Macbeth $\&$ Adrover, 2011). Con respecto a la vinculación entre la percepción gustativa y la olfativa, White y Prescott (2007) realizaron un experimento stroop intermodal en el que solicitaron a los participantes que identificaran el estímulo gustativo que se administraba junto con un estímulo olfativo. Según la presentación de los pares de estímulos gustativoolfativo se generaron tres condiciones: (1) congruencia: el estímulo olfativo y el estímulo gustativo pertenecían al mismo objeto; (2) incongruencia: el estímulo olfativo y el gustativo no pertenecían al mismo objeto y; (3) control: el estímulo olfativo era agua. Los resultados mostraron una facilitación en la identificación de los estímulos gustativos en la condición de congruencia, esto es, cuando el estímulo olfativo y el gustativo se referían al mismo objeto. Así, White y Prescott (2007) enfatizaron la interpretación del fenómeno en el significado adaptativo de los resultados debido a la importancia biológica de la discriminación rápida y precisa 
de los compuestos nutritivos en oposición a los compuestos potencialmente tóxicos antes de su consumo. En el mismo sentido, se ha destacado la vinculación anatómica entre las modalidades olfativa y gustativa a través del olfato retronasal (Razumiejczyk, Macbeth \& López Alonso, 2008). Adicionalmente, Razumiejczyk et al., (2012) estudiaron la interferencia stroop entre representaciones gustativas y auditivas. Los resultados mostraron que la interferencia fue menor en el nivel de estímulos congruentes, esto es, cuando el estímulo gustativo y la palabra presentada en forma auditiva coincidieron, en coherencia con lo observado por White y Prescott (2007). También se ha estudiado la interferencia stroop intermodal entre el gusto y la visión. Razumiejczyk et al. (2011) realizaron un experimento en el que administraron estímulos gustativos junto con imágenes fotográficas, y obtuvieron resultados similares a los de White y Prescott (2007) y Razumiejczyk et al. (2012). En todos los casos la interferencia en la memoria operativa resultó menor en el nivel de estímulos congruentes. Cuando la imagen pictórica y el estímulo gustativo se referían al mismo objeto, los sujetos tendían a identificar el estímulo gustativo de forma más rápida y eficaz. Razumiejczyk, Britos y Grigera Monteagudo (2010) obtuvieron resultados similares administrando estímulos gustativos junto con palabras escritas. Se compararon los datos obtenidos en ambos experimentos (Razumiejczyk et al., 2010, 2011) y los resultados mostraron que las palabras funcionaron como un mayor distractor que las imágenes en la tarea stroop intermodal necesitando un mayor tiempo de procesamiento para la identificación del estímulo gustativo administrado (Razumiejczyk et al., 2011). Se concluyó que los distractores lingüísticos producen una mayor competencia atencional con los estímulos gustativos que los distractores pictóricos. La relevancia de estos últimos resultados radica en la condición ecológica de los estímulos empleados. La infomación visual habitual puede tomar un formato tanto pictórico como lingüístico de palabras escritas.

En síntesis, el mayor tiempo de procesamiento obtenido en relación a la información lingüística se interpreta a partir de la complejidad en el procesamiento de este tipo de representaciones mentales de alto nivel. De este modo, la interferencia stroop intermodal entre el gusto y la visión es mayor con estímulos como palabras que con imágenes visuales debido a que el requerimiento atencional de las primeras es más arduo. La evidencia acumulada permite considerar que tal dificultad es el factor que explica las variaciones en la competencia entre los recursos cognitivos en la memoria operativa. Se sugiere por ello que el procesamiento habitual de las representaciones visuales lingüísticas forma parte del ajuste ecológico del individuo al ambiente. A partir de tal conjetura y sobre la base de los resultados obtenidos en estudios previos (Razumiejczyk et al., 2010, 2011, 2012; White \& Prescott, 2007) resulta relevante el estudio de la interferencia en la memoria operativa entre representaciones gustativas y visuales lingüísticas incompletas. Este estudio tiene como propósito extender la evaluación de la interferencia stroop intermodal entre el gusto y las palabras incompletas presentadas en forma visual. La hipótesis de trabajo aquí propuesta afirma que la omisión de letras en las palabras presentadas no genera procesos diferentes de los observados anteriormente para palabras completas y para formatos pictóricos. Se justifica esta hipótesis general por la tendencia a la adaptación observada en experimentos previos realizados sobre diferentes modalidades.

\section{Método}

\section{Participantes}

La muestra estuvo conformada por 42 participantes cuya edad promedio resultó de 24,43 ańos (de = 5,65 años). Participaron 27 mujeres $(64,3 \%)$ y 15 varones $(35,7 \%)$. Esta muestra se conformó mediante una invitación directa a estudiantes de grado matriculados en carreras de ciencias sociales de una universidad argentina. Las invitaciones se realizaron verbalmente antes del comienzo de una clase regular. Al igual que en estudios previos (Razumiejczyk, Macbeth \& Adrover, 2008; Razumiejczyk, Macbeth \& López Alonso, 2008) se determinaron los siguientes criterios de inclusión: 1) tener entre 20 y 40 años de edad (West, 2004); 2) ser no fumador y; 3) no haber ingerido ningún alimento ni bebida que no fuera agua durante las tres horas previas al experimento. En todos los casos la participación fue libre, voluntaria y con consentimiento informado. Los alumnos que aceptaron la invitación fueron convocados para formar parte del experimento en un día y horario específicos en las instalaciones de la misma universidad.

\section{Diseño}

Se empleó un diseño intra-sujetos de medidas repetidas para estudiar el efecto de tres tratamientos diferentes sobre la interferencia en memoria operativa de información 
gustativa y visual lingüística incompleta. Se determinó como variable independiente o factor a la congruencia del estímulo. Para este factor, se determinaron tres niveles en coherencia con estudios previos (Razumiejczyk et al., 2010; White \& Prescott, 2007): estímulos congruentes, estímulos incongruentes y estímulos controles. Los niveles del factor congruencia se generaron según la relación entre el estímulo gustativo y el estímulo visual incompleto administrados en el experimento: 1) estímulos congruentes: el estímulo visual incompleto coincide con el nombre completo del estímulo gustativo; 2) estímulos incongruentes: el estímulo visual incompleto no coincide con el nombre completo del estímulo gustativo pero representa una fruta; 3) estímulos controles: el estímulo visual incompleto y el estímulo gustativo no coinciden y el estímulo visual incompleto no representa un comestible. Se determinaron dos variables dependientes: el tiempo de reacción, medido en segundos con precisión de centésimos, y el número de aciertos.

\section{Materiales}

Se administraron como estímulos gustativos durazno, ciruela, fresa (llamada "frutilla" en Argentina) y naranja en forma de papilla licuada a temperatura ambiente. El coeficiente $\alpha$ de Cronbach para la identificación de estos estímulos resultó de 0,536 , lo cual sugiere una homogeneidad psicométrica adecuada (Razumiejczyk et al., 2010). Como estímulos visuales se presentaron en la pantalla de un ordenador palabras incompletas escritas en color negro sobre un fondo blanco. El criterio para producir cada palabra incompleta resultó de omitir al azar una letra por sílaba. En el caso de que la sílaba contara con una sola letra, no se omitía dicha letra. Como estímulos visuales congruentes se presentaron F_U_ILL_ (frutilla), D_R_ZN_(durazno), C_RU_L_ (ciruela) y N_RA_J_ (naranja), de este modo, el estímulo gustativo administrado coincidió con el estímulo visual léxico. Como estímulos visuales incongruentes se presentaron B_N__A (banana), AN__Á (ananá), SA_D_A (sandía) y MA_Z_ _A (manzana). Así, las palabras incompletas no coinciden con el estímulo gustativo administrado pero representan una fruta. Como estímulos controles se presentaron $M_{-} N_{-} D_{-}$ (moneda), A_T_OJ_S (anteojos), B_C__LET_(bicicleta) y R__OJ (reloj), esto es, las palabras incompletas no representan un alimento. Las palabras utilizadas en el experimento son de alta frecuencia en Argentina dado que nombran objetos y frutas típicas. La totalidad de los utensilios (cucharas, vasos y elementos de higiene) se desecharon luego de la utilización por cada participante.

\section{Hipótesis experimentales}

La hipótesis $H 1$ afirma que el tiempo de reacción $(T R)$ de los estímulos congruentes (CONG) es menor que el tiempo de reacción de los estímulos incongruentes $(I N C O N G)$ y controles (CONTROL). Formalmente, $H 1: \overline{T R}_{\text {INCONG }}>\overline{T R}_{\text {CONG }}<\overline{T R}_{\text {CONTROL }}$.Lahipótesis H2 afirma que el número de aciertos $(A)$ de los estímulos congruentes $(C O N G)$ es mayor que el número de aciertos de los estímulos incongruentes (INCONG) y controles $(C O N T R O L)$. Formalmente, $H 2: \bar{A}_{\text {INCONG }}<\bar{A}_{\text {CONG }}>\bar{A}_{\text {CONTROL }}$. Ambas hipótesis se justifican por los resultados obtenidos en estudios previos en los que la congruencia auditiva generó mayor número de aciertos y menor tiempo de reacción en la identificación gustativa (Razumiejczyk et al., 2012). Se halló esta misma tendencia en relación con representaciones gustativas y visuales pictóricas y visuales lingüísticas completas (Razumiejczyk et al., 2011). Asimismo, White y Prescott (2007) hallaron inicialmente los mismos resultados en relación con las modalidades olfativa y gustativa. Se espera encontrar la misma tendencia en relación con las modalidades gustativa y visual lingüística incompleta.

\section{Procedimiento}

El estudio fue realizado por dos experimentadores voluntarios; ellos recibieron una introducción relacionada con el procedimiento, pero desconocían su propósito. Se utilizó el paradigma de la tarea stroop intermodal (White \& Prescott, 2007). Se administraron a cada participante los estímulos gustativos junto con las palabras incompletas de manera simultánea. El experimentador entregaba en la mano del participante una cucharita descartable con el estímulo gustativo y éste se la llevaba a su boca en el momento en el que el experimentador lo indicaba. El experimentador presionaba una tecla de la computadora que hacía aparecer en la pantalla la palabra incompleta y comenzaba a medir el tiempo de respuesta. La consigna requería identificar el estímulo gustativo en el menor tiempo posible. Cuando el participante nombraba el estímulo gustativo administrado, el experimentador volvía a presionar la tecla de la computadora y así quedaba registrado el tiempo de reacción. Según la relación entre el estímulo gustativo y el estímulo visual se determinaron los tres niveles del factor congruencia (estímulos congruentes, incongruentes y controles). Se midió el tiempo de reacción y el número de aciertos para cada participante en cada ensayo. Se efectuaron 12 ensayos dado que se presentaron 4 estímulos gustativos y 3 niveles 
del factor congruencia. Se diseñó un dispositivo que impidió la observación del estímulo gustativo. Así, el participante sólo podía observar la pantalla de un ordenador durante el experimento. Se evitó de esta manera que la visión del color del estímulo influyere en el reconocimiento gustativo, en coherencia con las recomendaciones de Morrot, Brochet y Dubourdieu (2001). La Figura 1 presenta dos fotografías del dispositivo. Se acordó que antes de cada ensayo el participante efectuase una limpieza bucal con agua. Los pares de estímulos (gustativo y visual lingüístico incompleto) fueron administrados a cada participante en un orden aleatorio.
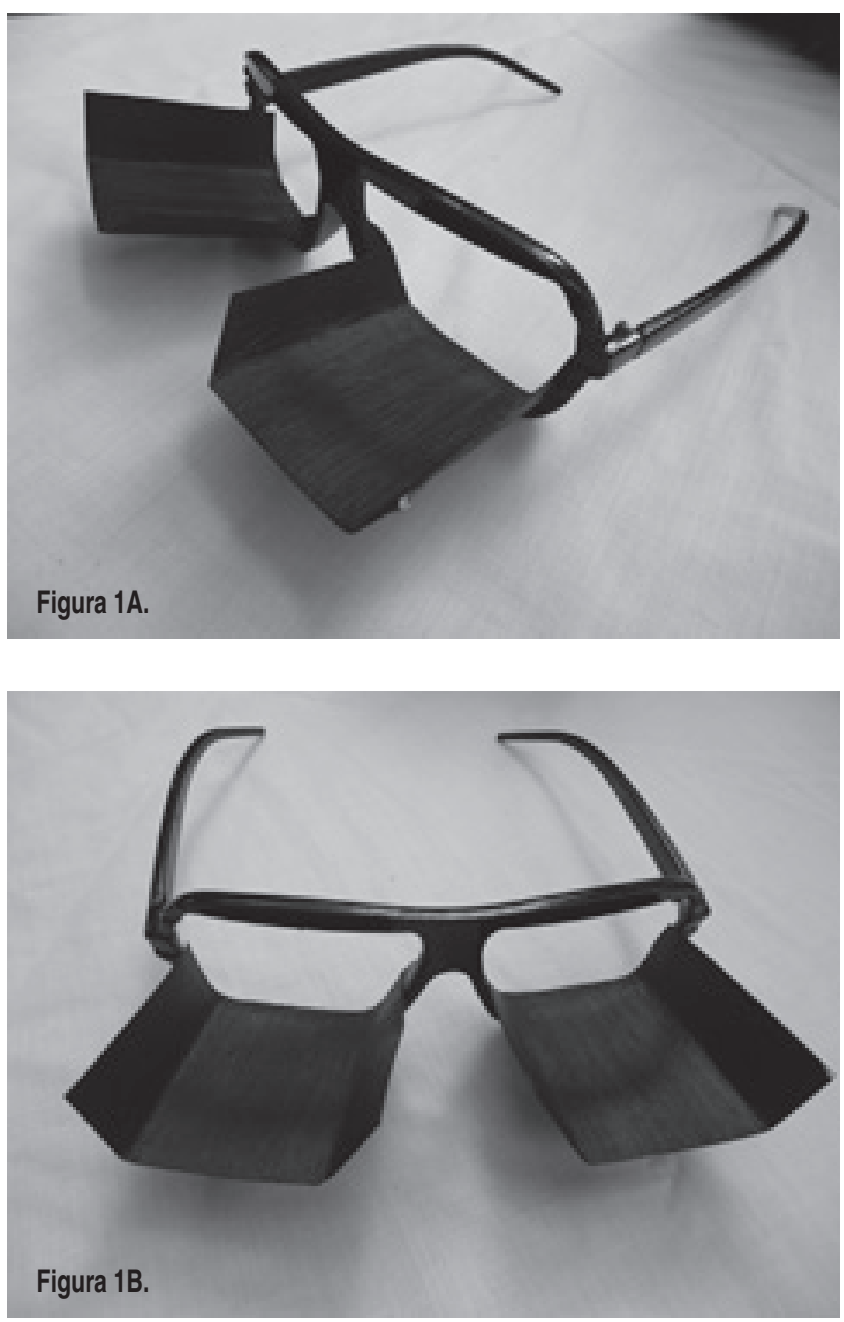

Figura 1. Dispositivo de reducción del campo visual.

Nota: Este dispositivo impide observar el estímulo gustativo durante su degustación, pero permite observar simultáneamente la pantalla del ordenador. Se construyó sobre la base de marcos para anteojos comunes sin cristales. Se añadieron cartones negros paralelos al piso de 6,5 centímetros en la parte frontal inferior. En estudios piloto y en experimentos previos se encontró que los participantes podían recibir los estímulos gustativos sin observar la cuchara de administración mediante el empleo de este dispositivo.

\section{Resultados}

En primer lugar, para poder tratar a la muestra como perteneciente a una misma población, se evaluaron posibles diferencias por género en las variables dependientes. No se hallaron diferencias significativas entre varones y mujeres entre los tres niveles del factor congruencia en las variables tiempo de reacción y número de aciertos, con tamaños del efecto próximos a cero $(d$ Cohen $<0,10)$. Se realizó un análisis de varianza intra-sujetos con el fin de comparar las diferencias del tiempo de reacción entre los tres niveles del factor congruencia. Se incluyeron todas las respuestas de los participantes en los tres niveles del factor congruencia para calcular las medias. Se encontró una diferencia estadísticamente significativa entre el tiempo de reacción de los estímulos congruentes, incongruentes y controles $\left(F(1,41)=22,496 ; p<0,01 ; \eta_{p}^{2}=0,354\right)$, en coherencia con la hipótesis H1. En comparaciones posteriores de a pares se encontró que el tiempo de reacción de los estímulos congruentes $(\bar{x}=6,36 ; d e=2,75)$ resultó menor $(t(41)=-3,518 ; p=0,001 ; d$ Cohen $=0,53)$ que el tiempo de reacción de los estímulos incongruentes $(\bar{x}=8,08 ; d e=3,67)$. El tiempo de reacción de los estímulos congruentes resultó menor $(t(41)=-4,743 ; p$ $<0,01 ; d$ Cohen $=0,85)$ que el tiempo de reacción de los estímulos controles ( $x=10,48 ; d e=6,2)$. Adicionalmente, el tiempo de reacción de los estímulos incongruentes resultó menor que el tiempo de reacción de los estímulos controles $(t(41)=-2,36 ; p=0,023 ; d$ Cohen $=0,47)$. En la Figura 2 a continuación se presenta una comparación visual de los tiempos de reacción para las tres condiciones. Se observa que la condición congruente es más rápida que la incongruente y que ésta es, a la vez, más rápida que la condición control.

El número de aciertos logrado por los participantes en los tres niveles del factor congruencia se estudió mediante un análisis de varianza intra-sujetos. Se encontró una diferencia estadísticamente significativa entre el número de aciertos de los estímulos congruentes, incongruentes y controles ( $F$ $\left.(1,41)=71,508 ; p<0,01 ; \eta_{p}^{2}=0,639\right)$, en coherencia con H2. En comparaciones posteriores de a pares se encontró que el número de aciertos de los estímulos congruentes $(x=3,12 ; d e=0,772)$ resultó mayor $(t(41)=7,873 ; p<$ 0,$01 ; d$ Cohen $=1,62$ ) que el número de aciertos de los estímulos incongruentes $(x=1,62 ; d e=1058)$. El número de aciertos de los estímulos congruentes resultó mayor $(t$ $(41)=8,456 ; p<0,01 ; d$ Cohen $=1,8)$ que el número de 


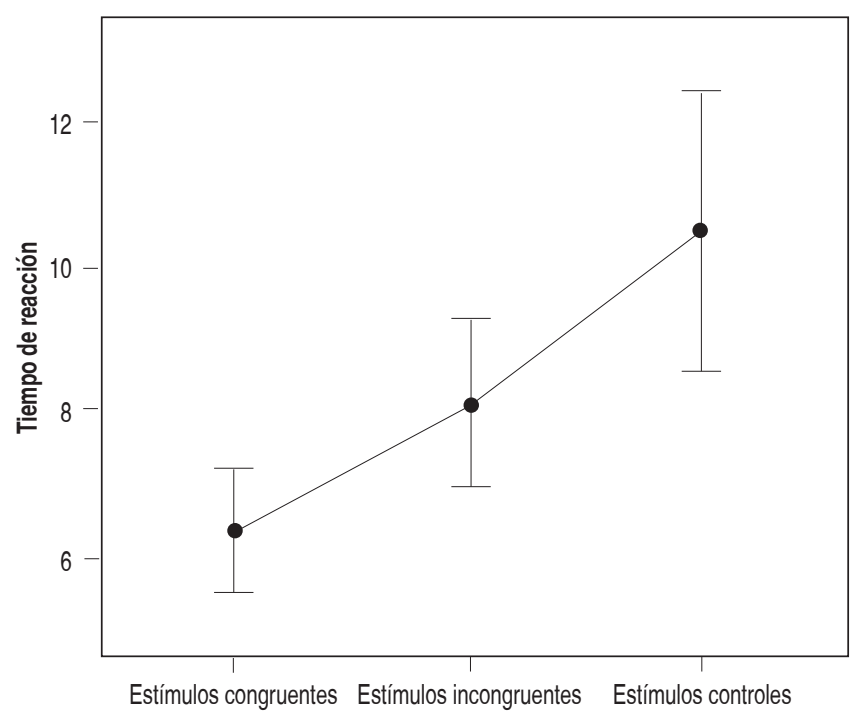

Figura 2. Comparación de medias del tiempo de reacción de los estímulos congruentes, incongruentes y controles.

Nota: La línea continua horizontal une puntos que representan las medias de tiempo de reacción en cada nivel del factor congruencia. Los segmentos verticales acotados que acompañan a cada punto representan el intervalo de confianza del $95 \%$ para cada media.

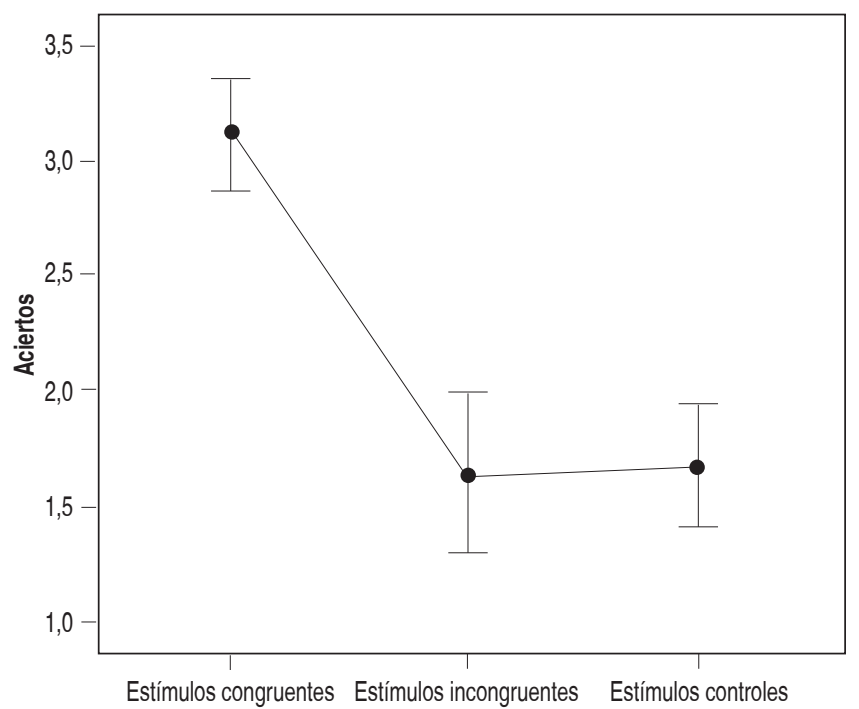

Figura 3. Comparación de medias del número de aciertos de los estímulos congruentes, incongruentes y controles.

Nota: Al igual que en la Figura 2, la línea continua horizontal une puntos que representan las medias de tiempo de reacción en cada nivel del factor congruencia. Los segmentos verticales acotados que acompañan a cada punto representan el intervalo de confianza del $95 \%$ para cada media. Se observa que los estímulos incongruentes y los controles se encuentran próximos entre sí y que los estímulos congruentes, en cambio, difieren significativamente tanto de los incongruentes como de los controles. aciertos de los estímulos controles $(\bar{x}=1,64 ; d e=0,879)$. No se encontraron diferencias significativas en el número de aciertos entre los estímulos incongruentes y los estímulos controles $(t(41)=-0,151 ; p=0,881 ; d$ Cohen $=0,02)$. La Figura 3 muestra la media del número de aciertos de los estímulos congruentes, incongruentes y controles. Todas las variables comparadas resultaron normales y homocedásticas por las pruebas de Kolmogorov-Smirnov y Levene, respectivamente, por lo cual se aplicaron pruebas paramétricas para contrastar las hipótesis $\mathrm{H} 1$ y $\mathrm{H} 2$ dentro del modelo lineal.

\section{Discusión}

Se ha estudiado la interferencia entre las representaciones gustativas y las representaciones visuales lingüísticas incompletas en la memoria operativa a través de la tarea stroop intermodal. Los resultados muestran que la interferencia stroop intermodal resultó menor en el nivel de estímulos congruentes, produciendo mayores aciertos y menores tiempos de reacción con tamaño del efecto grande y mediano respectivamente cuando el estímulo gustativo y el objeto al que se refería la palabra incompleta presentada en forma visual coincidieron. Estos resultados sugieren que durante el procesamiento de estímulos congruentes la competencia atencional entre los estímulos gustativos y visuales es mínima posiblemente debido a que la información converge. Aquí, el conflicto del procesamiento cognitivo es reducido aún a pesar de las letras faltantes en el estímulo visual presentado en forma de palabra escrita. En este sentido, la evidencia sugiere que se produciría una identificación eficiente de la palabra incompleta. Es decir, se propone que la tarea stroop del experimento propuesto en este estudio requiere la activación de dos procesos en competencia (Cho et al., 2006; Kahneman \& Chajczyk, 1983; Kim et al., 2008; Mitterer et al., 2003). La identificación del estímulo gustativo, entendida como proceso explícito, compite con la identificación de la palabra incompleta, entendida como consigna implícita. La segunda tarea es implícita porque no se incluye en las instrucciones del experimento dadas a los participantes, a quienes se les indica solamente que observen la pantalla del ordenador. En relación con esta doble tarea, la evidencia acumulada en múltiples experimentos sugiere que el procesamiento de las claves lingüísticas resulta automático (Fodor, 1983). Los hallazgos del presente estudio son compatibles con tales antecedentes. 
El principal aporte del presente estudio stroop se refiere al ajuste logrado por los participantes ante una tarea experimental que provee información ecológica incompleta, esto es, los participantes debieron procesar en primer lugar el significado de las palabras con letras faltantes para pasar luego al procesamiento gustativo. El diseño implementado se centró en la congruencia de los estímulos como variable independiente. Por ejemplo, en el caso del nivel de incongruencia, cuando el participante recibe el estímulo gustativo de fresa (frutilla) mientras que simultáneamente se presenta en el ordenador la palabra incompleta "MA_Z__A" (manzana) como estímulo visual, los patrones de acierto y tiempo de reacción dependen solamente de la incongruencia y no del llenado mental implícito de las letras omitidas. Así, se produce un mayor tiempo de reacción y se comenten mayores errores que cuando frente al mismo estímulo gustativo se presenta el estímulo visual "F_U_ILL_" (frutilla) como estímulo congruente. De este modo, la interferencia stroop es mayor en el nivel de estímulos incongruentes debido a la manipulación de la presentación de estímulos, esto es, de la coincidencia o no entre el estímulo gustativo administrado y la palabra incompleta visual (estímulos congruentes, estímulos incongruentes, estímulos controles). Así, las diferencias encontradas en las variables dependientes son atribuibles solamente a la manipulación de la variable independiente y no al procesamiento adicional de las palabras incompletas.

En coherencia con estudios previos, en el nivel de estímulos incongruentes y controles los resultados sugieren que los participantes no lograron inhibir los distractores lingüísticos incompletos visuales para obtener las respuestas relativas a la identificación de los estímulos gustativos de forma rápida y eficaz (White \& Prescott, 2007). Estos resultados son compatibles con los hallados por Razumiejczyk et al. (2011) en relación con representaciones lingüísticas completas. Cuando una tarea stroop con estímulos gustativos involucra el lenguaje, la integración intermodal gustativa y visual se vuelve sofisticada. Este mismo aspecto del stroop fue advertido por Razumiejczyk et al. (2012) en las modalidades gustativa y auditiva. Sobre la base de los resultados aquí obtenidos resulta relevante realizar en el futuro un estudio factorial para comparar estímulos auditivos, visuales completos y visuales incompletos con el gusto. Asimismo, en futuras investigaciones se propone estudiar la interferencia stroop entre representaciones gustativas y visuales con otras variedades de estímulos lingüísticos (por ejemplo anagramas). Los datos aquí hallados son consistentes y extienden los resultados hallados por White y Prescott (2007), quienes estudiaron la interferencia stroop entre las representaciones gustativas y olfativas.

Un resultado inesperado del presente estudio consiste en la relación entre el procesamiento de estímulos incongruentes y controles. Por implicar mayor proximidad semántica intermodal, resulta esperable que los estímulos incongruentes consuman mayor tiempo y generen más errores que los estímulos controles. Se encontró, por el contrario, que los incongruentes se procesaron más rápido que los controles pero no con mayor éxito. La evidencia generada en este experimento no permite sugerir una explicación con fundamento empírico para este resultado. Es necesario realizar nuevos experimentos para analizar de manera controlada este fenómeno. A manera de ejemplo, se sugiere para futuras investigaciones realizar un estudio de gradiente semántico con por lo menos dos niveles de estímulos incongruentes según la distancia semántica entre la palabra del estímulo incongruente y el estímulo gustativo. Por ejemplo, para el estímulo gustativo de durazno, el estímulo visual léxico más conflictivo podría ser damasco, dado que se parece tanto en el sabor como en su aspecto visual. De este modo se podrían comparar los tiempos de reacción y los números de aciertos entre los estímulos controles y dos niveles de estímulos incongruentes.

A partir de los resultados obtenidos, se podría argumentar que éstos muestran sólo un efecto léxico. Es decir, que los participantes consideraron solamente la palabra incompleta presentada en forma visual para dar sus respuestas. El problema con esta objeción radica en que supone la capacidad de inhibición voluntaria del canal sensorial gustativo, a pesar de ser requerido por la consigna de la tarea experimental. Tal capacidad de inhibición voluntaria resulta poco probable según la evidencia acumulada por el paradigma intermodal empleado en este estudio. En múltiples experimentos intermodales se ha encontrado que la modalidad complementaria a la considerada por la tarea experimental se procesa de manera inevitable (White \& Prescott, 2007). Así, la reducción de los hallazgos del presente estudio a un efecto léxico parece poco probable.

Sin embargo, es posible que en el procesamiento de los estímulos congruentes e incongruentes se haya producido una aceleración en los tiempos de reacción por efecto léxico. Quizás este mismo efecto pueda explicar el retraso 
antes mencionado de los controles en comparación con los incongruentes. En cualquier caso, no parece verosímil que en este y otros estudios del paradigma intermodal los participantes sean capaces de inhibir voluntariamente un canal sensorial y responder sólo a partir del complementario. Por el contrario, la evidencia acumulada del paradigma stroop sugiere la presencia de interferencia (Cho et al., 2006; Kahneman \& Chajczyk, 1983; Kim et al., 2008; Mitterer et al., 2003).

Una limitación del presente estudio consiste en el empleo de una selección específica de estímulos gustativos. Se recomienda ampliar tal selección para incluir nuevos estímulos que cumplan las mismas condiciones de validación que los aquí utilizados (Razumiejczyk et al., 2012). Una ventaja de este estudio consiste en la condición natural o ecológica de los materiales empleados. Se administraron papillas de frutas a temperatura ambiente, a diferencia de otros estudios que utilizaron estímulos artificiales en forma de esencias o de estimulación eléctrica sobre la lengua (Halpern, 2005; Keast, Canty \& Breslin, 2004; Kelling \& Halpern, 1987; Kobayakawa, Ogawa, Kaneda, Ayabe-Kanamura \& Saito, 1999; Schoenfeld, et al., 2004; Yamamoto et al., 2003). Todos los estímulos empleados en el presente estudio pertenecen a la dieta habitual de los participantes. La importancia de estos resultados radica en la evidencia de adaptación del organismo al ambiente en las tareas específicas estudiadas (Pinker, 1997). Una interpretación evolucionista de este hallazgo permite sugerir que la identificación rápida y acertada de los estímulos congruentes se corresponde con la adaptación eficiente al ambiente. En el mismo sentido, resulta razonable que la incongruencia requiera mayor procesamiento e incurra en mayores errores de identificación. Es en tal contexto que se procuró en este estudio que el diseño experimental se ajustase a la relación natural que posee el individuo con el ambiente en el cual se desenvuelve; por esta razón se utilizaron estímulos gustativos naturales y se consideró que las palabras son estímulos visuales habituales en el nicho ecológico del individuo. El tamaño del efecto obtenido para la interferencia stroop intermodal tiende a ser grande ( $d$ Cohen $>0,80$ ), lo cual sugiere que los participantes del experimento se encuentran bien adaptados al ambiente en el cual se desempeñan habitualmente (Razumiejczyk \& Jáuregui, 2012).

\section{Referencias}

Baddeley, A. (1995). Working memory: the interface between memory and cognition. En D. L. Schacter \& E. Tulving (Eds.) Memory systems 1994 (pp. 351368). Cambridge: The MIT Press.

Banich, M. T., Milham, M. P., Atchley, R., Cohen, N. J., Webb, A., Wszalek, T., Kramer, A. F., Liang, Z. P., Wright, A., Shenker, J. \& Magin, R. (2000). fMRI studies of stroop tasks reveal unique roles of anterior and posterior brain systems in attentional selection. Journal of Cognitive Neuroscience, 12, 988-1000. doi; $10.1162 / 08989290051137521$

Cho, Y. S., Lien, M. C. \& Proctor, R. W. (2006). Stroop dilution depends on the nature of the color carrier but not on its location. Journal of Experimental Psychology: Human Perception \& Performance, 32, 826-839. doi: 10.1037/0096-1523.32.4.826

Fodor, J. A. (1983). Modularity of mind: An essay on faculty psychology. Cambridge, Mass: MIT Press.

Halpern, B.P. (2005). Temporal characteristics of human taste judgements as calibrations for gustatory event-related potentials and gustatory magnetoencephalographs. Chemical Senses, 30, 228-234. doi: 10.1093/chemse/ bjh 197

Kahneman, D. \& Chajczyk, D. (1983). Tests of the automaticity of reading: dilution of stroop effects by color-irrelevant stimuli. Journal of Experimental Psychology: Human Perception \& Performance, 9, 497-509. doi: 10.1037/0096-1523.9.4.497

Keast, R.S.J., Canty, T.M. \& Breslin, P.A.S. (2004). Oral zinc sulphate solutions inhibit sweet taste perception. Chemical Senses, 26, 513-521. doi: 10.1093/chemse/ bjh053

Kelling, S.T. \& Halpern, B.P. (1987). Taste judgements and gustatory stimulus duration: simple taste reaction times. Chemical Senses, 12, 543-562. doi: 10.1093/ chemse/ 12.4.543

Kim, H., Cho, Y. S., Yamaguchi, M. \& Proctor, R. W. (2008). Influence of color availability on the stroop color-naming effect. Perception \& Psychophysics, 70, 1540-1551. doi: 10.3758/PP.70.8.1540

Kirn, S. Y., Kirn, M. S. \& Chun, M. M. (2005). Concurrent working memory load can reduce distraction. Proccedings of National Academy of Sciences of the United States of America, 102, 16524-16529. doi: $10.1073 / 0505454102$ 
Kobayakawa, T., Ogawa, H., Kaneda, H., Ayabe-Kanamura, S. \& Saito, S. (1999). Spatio-temporal analysis of cortical activity evoked by gustatory stimulation in humans. Chemical Senses, 24, 201-209. doi: $10.1093 /$ chemse/24.2.201

MacLeod, C. M. (1991). Half a century of research on the stroop effect: An interactive review. Psychological Bulletin, 109(2), 163-203.

MacLeod, C. M. (1992). The stroop task: The "gold standard" of attentional measures. Journal of Experimental Psychology, 121, 12-14. doi: 10.1037/00963445.121.1.12

Mitterer, H., La Heij, W. \& Van der Heijden, A. H. C. (2003). Stroop dilution but not word- processing dilution: evidence for attention capture. Psychological Research, 67, 30-42.

Morrot, G., Brochet, F. \& Dubourdieu, D, (2001). The color of odors. Brain Language, 79, 309-320. http:// dx.doi.org/10.1006/brln.2001.2493

Pinker, S. (1997). How the mind works. New York, NY: Norton.

Prescott, J., Johnstone, V. \& Francis, J. (2004). Odortaste interactions: effects of attentional strategies during exposure. Chemical Senses, 29, 331-340. doi: 10.1093/chemse/bjh036

Razumiejczyk, E., Macbeth, G. \& Adrover, J. F. (2008). Priming intramodal e intermodal: un estudio gustativo-visual. Revista de investigación en psicología, 11(1), 69-79.

Razumiejczyk, E., Macbeth, G. y López Alonso, A. O. (2008). Evidencia de priming gustativo y su disociación con la memoria explícita. Revista Universitas Psychologica, 7(2), 549-556.

Razumiejczyk, E., Britos, P. y Grigera Monteagudo D. (2010). Interferencia entre representaciones gustativas y lingüísticas en la memoria operativa: evidencia de la tarea stroop intermodal. Calidad de vida, 5, 83-90.

Razumiejczyk, E., Bacci, C., Iriarte, M.P., Britos, P., Genovese, I., Grigera Monteagudo, D., Caselli, G. y Bellucci, P. (2010). Selección de estímulos para el estudio de los procesos cognitivos relacionados con la modalidad gustativa. Psicología y Psicopedagogía, 23.

Razumiejczyk, E. y Jáuregui, M. (2012). Estudios intermodales: revisión de antecedentes. Revista Psicología y Psicopedagogía, 28.

Razumiejczyk, E., Jáuregui, M. \& Macbeth, G. (2012). Interferencia stroop intermodal entre representaciones gustativas y auditivas. Revista CES Psicología, 5(2), 25-41.
Razumiejczyk, E., Macbeth, G. y Adrover, J. F. (2011). Comparación de la interferencia stroop intermodal entre representaciones gustativas y visuales por imágenes y por palabras. Boletín de Psicología, 101, 7-20.

Razumiejczyk, E. Macbeth, G. y López Alonso, A. O. (2008). La vinculación entre las modalidades gustativa y olfativa en el reconocimiento del sabor. Psico Logos, 17, 5-12.

Rolls, E. T. (2004). Multisensory neuronal convergence of taste, somatonsensory, visual, olfactory, and auditory inputs. En G. A. Calvert, C. Spence \& B. E. Stein (Eds.) The Handbook of Multisensory Processes. (pp 311-331). Cambridge, MA.: MIT Press.

Schoenfeld, M. A., Neuer, G., Tempelmann, C., Schubler, K., Noesselt, T., Hopf, J-M. \& Heinze, H-J. (2004) Funtional magnetic resonance tomography correlates of taste perception in human primary paste cortex. Neurocience, 127, 347-353. http:// dx.doi.org/10.1016/j.neuroscience.2004.05.024

Sreenivasan, K. K. \& Jha, A. P. (2007). Selective attention supports working memory maintenance by modulating perceptual processing of distractors. Journal of Cognitive Neuroscience, 19, 32-41. doi:10.1162/jocn.2007.19.1.32

Stevenson, R. J. \& Boakes, R. A. (2004). Sweet and sour smells: learned synesthesia between the senses of taste and smell. En G. A. Calvert, SC. Spence \& B. E. Stein (Eds.) The Handbook of Multisensory Processes (pp 69-83). Cambridge, MA: M.I.T. Press.

Stroop, J. R. (1935). Studies of interference in serial verbal reactions. Journal of Experimental Psychology, 8, 643-666. doi: 10.1037/h0054651

West, R. (2004). The effects of aging on controlled attention and conflict processing in the stroop task. Journal of Cognitive Neuroscience, 16, 103-113. doi:10.1162/089892904322755593

White, T. L. \& Prescott, J. (2007). Chemosensory crossmodal stroop effects: congruent odors facilitate taste identification. Chemical Senses, 32, 337-341. doi: 10.1093/chemse/bjm001

Yamamoto, C., Takehara, S., Morikawa, K., Nakagawa, M., Yamaguchi, M., Iwaki, S., Tonoike, M. \& Yamamoto, T. (2003). Magnetoencephalographic study of cortical activity evoked by electrogustatory stimuli. Chemical Senses, 28, 245-251. doi: 10.1093/ chemse/28.3.245 
